\title{
Association between macronutrients and fibre with circulating Insulin-Like Growth Factor-I in the UK Biobank
}

\author{
C.Z. Watling ${ }^{1}$, R.K. Kelly ${ }^{1}$, T.Y.N. Tong ${ }^{1}$, G. Fensom ${ }^{1}$, C. Piernas ${ }^{2}$, E.L. Watts ${ }^{1}$, S. Tin Tin $^{1}$, \\ A. Knuppel ${ }^{1}$, J. Schmidt ${ }^{1}$, R.C. Travis ${ }^{1}$, T.J. Key ${ }^{1}$ and A. Perez-Cornago ${ }^{1}$ \\ ${ }^{1}$ Cancer Epidemiology Unit, Nuffield Department of Population Health, University of Oxford. Oxford, United Kingdom \\ and \\ ${ }^{2}$ Nuffield Department of Primary Care, University of Oxford. Oxford, United Kingdom
}

Circulating Insulin-Like Growth Factor I (IGF-I) has been associated with higher risk of several common types of cancer, such as prostate, breast, and colorectal cancer ${ }^{(1)}$, and dietary intake has been suggested to influence IGF-I concentrations ${ }^{(2)}$. However, previous observational studies investigating the association of nutrients with circulating IGF-I have been limited by small sample sizes. We assessed the association of macronutrients and fibre intake with circulating IGF-I concentrations in an observational analysis in the UK Biobank; a large cohort of British adults.

In this analysis, participants were selected if they completed at least four (maximum of five) web-based 24-hour dietary assessments ${ }^{3}$ and had serum IGF-I measured $(\mathrm{n}=12,000)$. Usual macronutrient and fibre intakes were determined by the mean of completed 24-hour dietary assessments. Multivariable linear regression was used to assess the associations of these dietary factors with circulating IGF-I. Sensitivity analyses were conducted in participants who had a second blood sample five years after recruitment, using the mean of the two IGF-I measurements $(n=2,581)$.

The mean circulating IGF-I concentration was $21.96 \mathrm{nmol} / \mathrm{L}$. Consumption of $2.5 \%$ higher energy intake from total protein, dairy protein, milk protein, and yogurt protein were associated with 0.57 (95\% confidence interval (CI): 0.47-0.66), 0.71 (95\% CI:0.500.92), 1.17 (95\% CI:0.87-1.48), and 1.33 (95\% CI:0.80-1.85) nmol/L higher circulating concentrations of IGF-I respectively, whereas cheese protein was not associated with IGF-I concentration. A 5 gram/day higher intake of fibre was associated with $0.46(95 \%$ CI:0.35-0.57) nmol/L higher concentration of IGF-I. When analyses were restricted to participants with two blood samples, results remained largely the same, with milk protein being most strongly associated with the average measurements of IGF-I concentration. Carbohydrates and fats were not materially associated with IGF-I concentrations.

We found that dairy protein was the macronutrient most strongly associated with circulating IGF-I concentrations. However, this association varied by dairy protein source, with the strongest association being for milk and yogurt protein. Moreover, dietary fibre intake was also positively associated with IGF-I, which warrants further investigation.

1. Pollak M (2012) Nat Rev Cancer 12, 3, 159-169.

2. Crowe FL, Key TJ, Allen NE et al. (2009) Cancer Epidemiol Biomarkers and Prev, 18, 3, 1333-1440.

3. Liu B, Young H, Crowe FL, et al. (2011) Public Health Nutr 14, 11, 1998-2005. 